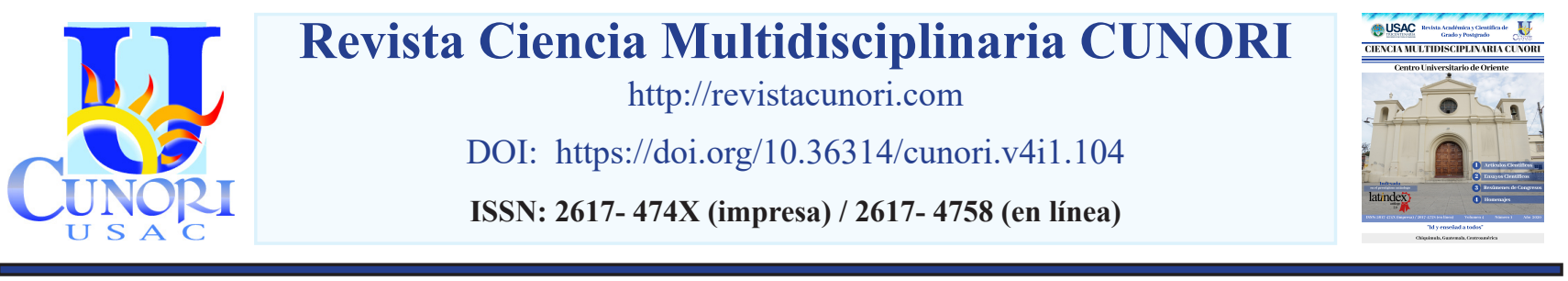

Como citar el artículo

Hernández, E., Vides, I. y Retana, R., (2020). Caracterización de pacientes y evolución de la cirugía para corrección de transposición de grandes arterias. Revista Ciencia Multidisciplinaria CUNORI, 4(1), 7-14. DOI: https://doi.org/10.36314/cunori.v4i1.104

\title{
Caracterización de pacientes y evolución de la cirugía para corrección de transpo- sición de grandes arterias
}

\section{Characterization of patients and evolution of the surgery for correction of transposition of great arteries}

Eliú Hernández*, Iván Vides \& Ronaldo Retana

Centro Universitario de Oriente (CUNORI), Universidad de San Carlos de Guatemala

Recibido: 02 de junio de 2019 / Revisión: 31 de julio de 2019 / Aceptado: 04 de febrero de 2020

Disponible en internet el 29 de mayo de 2020

*Autor para correspondencia.

Correo electrónico: carloseliu38@gmail.com

\section{Resumen}

【 a transposición de grandes arterias (TGA) es una de las principales cardiopatías congénitas cianógenas, la cual sin tratamiento Luirúrgico representa una alta tasa de mortalidad, siendo el switch arterial la cirugía de elección. Caracterizar a los pacientes sometidos a cirugía para corrección de transposición de grandes arterias y su evolución. El estudio descriptivo retrospectivo en donde se revisaron los archivos de 26 pacientes que fueron sometidos a cirugía para corrección de transposición de grandes arterias durante el periodo de enero de 2014 a diciembre de 2018 en la sección pediátrica de la unidad de cirugía cardiovascular de Guatemala, los datos se analizaron utilizando estadística descriptiva. Se determinó que el 58\% de los pacientes son de sexo masculino, el peso varía entre $2.5-5 \mathrm{~kg}$ en el $62 \%$ de los casos, la edad a la que fueron sometidos a cirugía fue menor de 10 días en el $40 \%$ de los casos, el procedimiento quirúrgico de elección fue el switch arterial en el 80\% de los casos. La mortalidad global fue del 31\%, si se excluyen las muertes de origen no cardiaco, la mortalidad baja a 19\%. La cirugía de elección sigue siendo el switch arterial, el equipo médico quirúrgico ha adquirido más experiencia al correr de los años, al haber logrado disminuir la mortalidad por origen cardiaco después de cirugía hasta el 19\%.

Palabras clave: cardiopatía congénita, transposición de grandes arterias, switch arterial

\section{Abstract}

$\mathrm{T}$ ransposition of the great arteries (TGA) is one of the main cyanogenic congenital heart diseases, which without surgical treat-

ment represents a high mortality rate, with the arterial switch being the surgery of choice. Characterize patients undergoing surgery to correct transposition of the great arteries and their evolution. The retrospective descriptive study where the files of 26 patients who underwent surgery to correct transposition of the great arteries during the period from January 2014 to December 2018 in the pediatric section of the cardiovascular surgery unit in Guatemala, were reviewed. Data were analyzed using descriptive statistics. It was determined that $58 \%$ of the patients are male, the weight varies between $2.5-5 \mathrm{~kg}$ in $62 \%$ of the cases, the age at which they underwent surgery was less than 10 days in $40 \%$ of the patients. cases, the surgical procedure of choice was the arterial switch in $80 \%$ of the cases. Overall mortality was $31 \%$, if non-cardiac deaths are excluded, mortality drops to $19 \%$. The surgery of choice continues to be the arterial switch, the surgical medical team has gained more experience over the years, having reduced mortality from cardiac origin after surgery to $19 \%$.

Keywords: congenital heart disease, transposition of great arteries, arterial switch 


\section{Introducción}

La transposición de grandes arterias (TGA) es la cardiopatía congénita cianógena más frecuente, en donde la aorta sale del ventrículo morfológico derecho y la arteria pulmonar sale del ventrículo morfológico izquierdo, lo cual provoca que la sangre sistémica que no contiene oxigeno regrese por medio de la aorta al circuito sistémico; y que la sangre pulmonar que contiene oxígeno, regrese al circuito pulmonar (Vélez \& Echeverri 2003).

Generalmente estos pacientes se presentan con cianosis leve-moderada, se auscultan soplos patológicos a nivel cardiaco, o presentan algún tipo de trastorno dismorfogenético que se asocie a cardiopatía congénita (Ronderos Dumit, 2010).

Los pacientes con cardiopatías congénitas necesitan centros de atención especializados en el diagnóstico y tratamiento oportuno (Dip et al. 2011). En Guatemala existe la sección pediátrica de la unidad de cirugía cardiovascular desde 1997, la cual ha ayudado en el diagnóstico y tratamiento de miles de niños (UNICAR, 2018).

Representa un reto a todo nivel, y en países en vías de desarrollo, este reto es mucho mayor. La mortalidad es alta, teniendo mucho que ver el diagnostico tardía. En Guatemala se revisaron los casos tratados en la sección pediátrica de la unidad de cirugia cardiovascular de Guatemala tratados hasta el 2009 y se evidencio que la mortalidad tras cirugía era del 47\% (Leon-Wyss et al. 2011).

Por lo anterior se realizó una revisión de los casos tratados durante los últimos 5 años, buscando caracterizar a los pacientes que fueron sometidos a cirugía para corrección de transposición de grandes arterias y su evolución.

\section{Materiales y métodos}

Se realizó un estudio descriptivo retrospectivo sobre las características y la evolución de los pacientes que fueron sometidos a cirugía para corrección de transposición de grandes arterias en la sección pediátrica de la unidad de cirugía cardiovascular de Guatemala durante enero de 2014 a diciembre de 2018. Durante dicho periodo fueron sometidos a cirugía 33 pacientes, de los cuales 5 archivos médicos se extraviaron y a 2 pacientes se les realizo cirugía paliativa (septectomía) por lo que se revisaron los archivos de 26 pacientes.

\section{Resultados}

Con respecto a las características de los pacientes el sexo masculino predominó con el 58\% (15) de los casos y el resto fueron de sexo femenino $42 \%(11)$. De los 26 pacientes el 61\% (16) pesó entre 2.5-5 $\mathrm{kg}$. La edad de referencia fue menor de 10 días en el $50 \%$ (promedio 16 días) y la edad al momento del procedimiento quirúrgico fue menor de 10 días en el 40\% (promedio 17 días). Los hospitales que más pacientes refirieron fueron Instituto Guatemalteco de Seguridad Social, Hospital Roosevelt y Hospital San Juan de Dios en el 50\% de los casos (13), el otro 50\% fueron referidos por hospitales del interior del país. 
El tipo de transposición de grandes arterias más frecuente fue la transposición de grandes arterias con septo intacto en $73 \%$ (19) y que la cirugía más empleada para la corrección de transposición de grandes arterias fue el Switch arterial en el $80 \%$ de los casos.

El nivel de complejidad de acuerdo al procedimiento quirúrgico según la escala de estratificación de riesgo Aristóteles fue de 4/4 para el Switch arterial, Switch arterial más corrección de comunicación interventricular y procedimiento Rastelli; y complejidad 3/4 para el procedimiento de Senning.

De acuerdo a la evolución el tiempo de estadía en la unidad de cuidados intensivos cardiovasculares pediátricos fue mayor de 10 días en el $65 \%$ (promedio 14 días) y el tiempo de estadía intrahospitalario fue entre 10-20 días en el 54\% (promedio 20 días).

Se utilizó arresto circulatorio en hipotermia profunda en 1 paciente pues además de la transposición de grandes arterias tenia hipoplasia del arco aórtico, lo que representa el $4 \%$ de los casos.

La mortalidad global fue de 31\% (8), la mortalidad por causa cardiaca es del 19\%. La mortalidad esperada según la escala de estratificación de riesgo Aristóteles es del 3-4\%. La causa de defunción fue falla ventricular en el $62 \%$ de los casos (5) y choque séptico en el 38\% de los casos (3).

Figura 1. Características de acuerdo al sexo de los pacientes sometidos a cirugía.

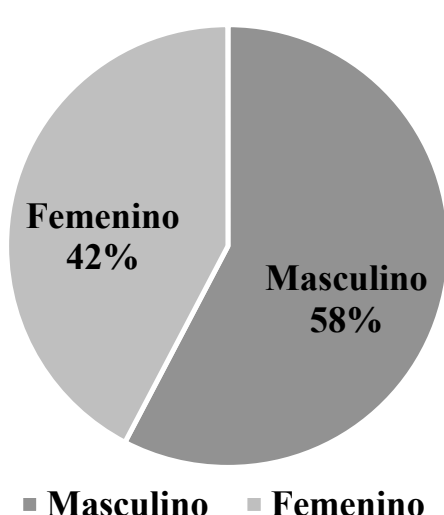

$n=26$

Boleta de recolección de datos 2019 
Figura 2. Caracterización de pacientes de acuerdo a la edad de referencia

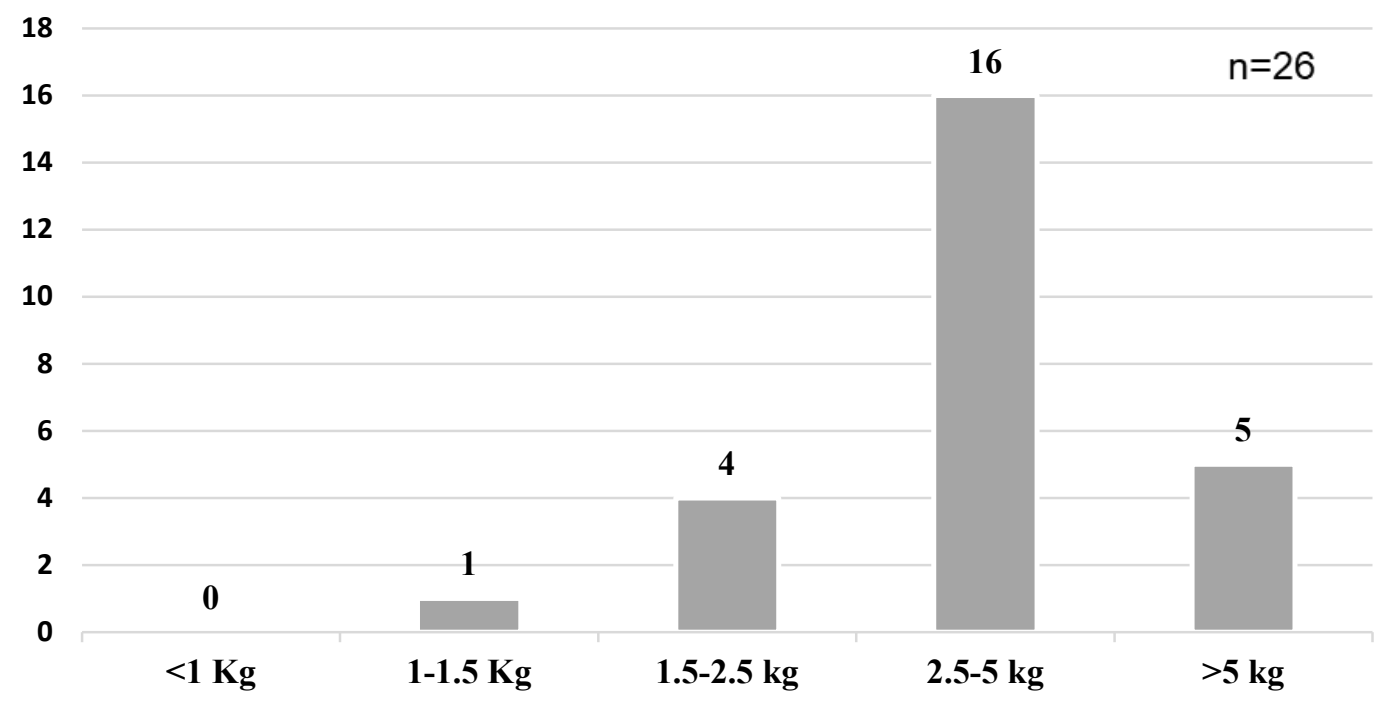

Boleta de recolección de datos 2019

Figura 3. Caracterización de acuerdo al tipo de transposición de grandes arterias y al tipo de cirugía utilizada para su corrección.

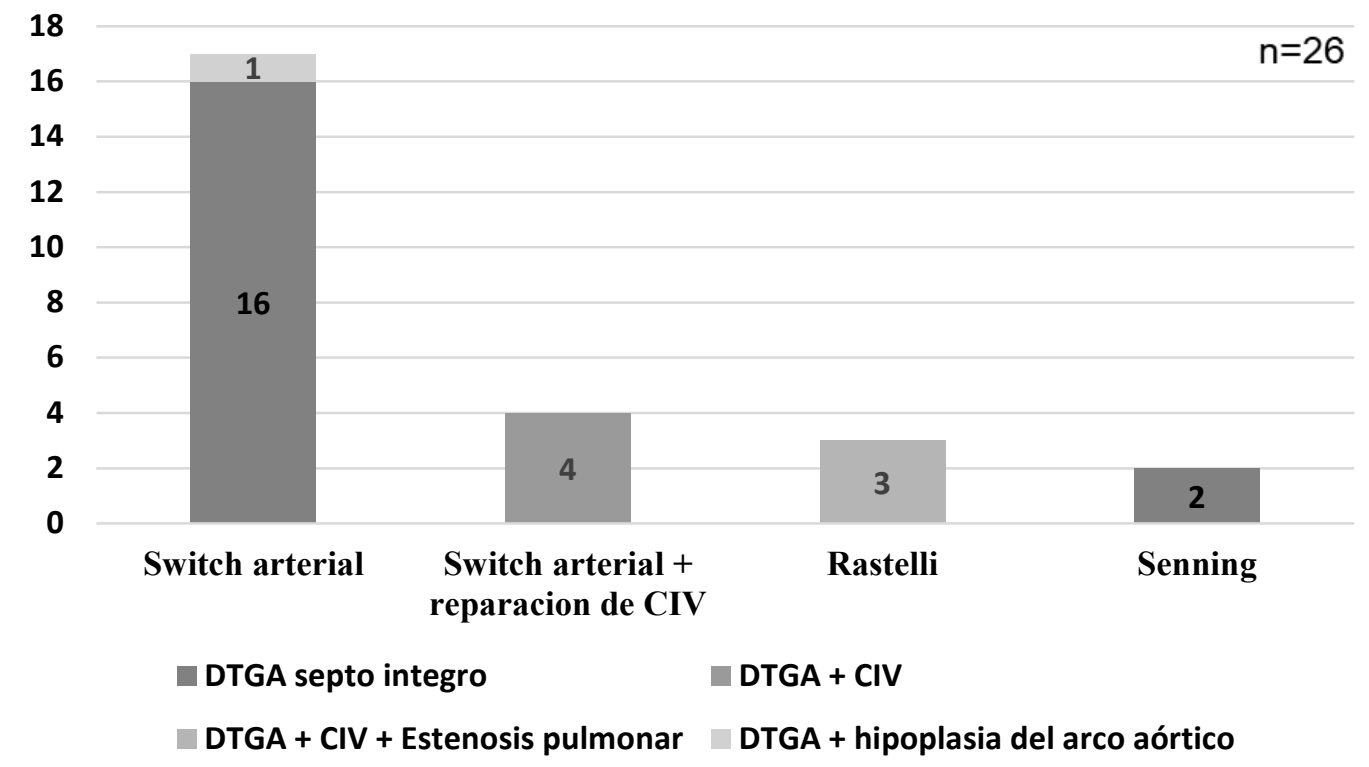

Boleta de recolección de datos 2019 
Figura 4. Caracterización de acuerdo a mortalidad de los pacientes sometidos a cirugía para corrección de transposición de grandes arterias.

I

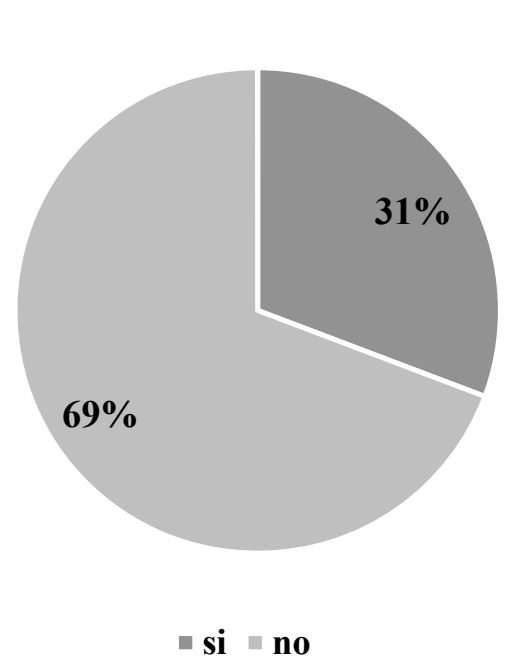

Boleta de recolección de datos 2019

Figura 5. Caracterización de acuerdo a la causa de defunción.

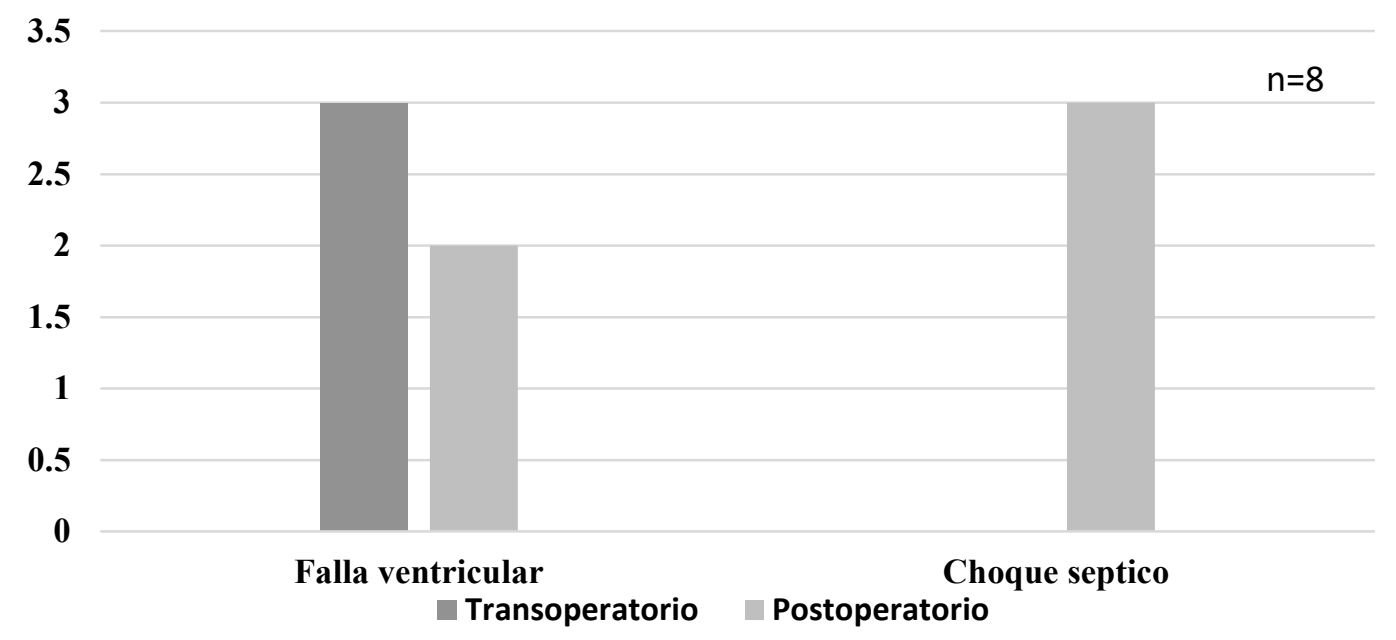

Boleta de recolección de datos 2019.

\section{Discusión}

Se caracterizó a los pacientes de acuerdo al sexo, evidenciado que el 58\% de los pacientes fueron de sexo masculino y el $42 \%$ fueron de sexo femenino lo cual concuerda con los datos obtenidos a nivel internacional en donde se reportan más casos en el sexo masculino, aunque no se ha reportado que existe predominio absoluto en algún sexo. Se evidenció una media de edad de 17 días al momento de la cirugía, aunque existen dos datos aislados de pacientes que fueron sometidos a cirugía al año cuatro meses y dos años respectivamente. 
Con respecto al peso de los pacientes el 61\% (16) pesaban entre 2.5-5 $\mathrm{kg}$ con una media de peso de 3.5 $\mathrm{kg}$, aun así se intervino a 1 paciente que pesaba menos de $1.5 \mathrm{~kg}$, lo cual representa un verdadero reto quirúrgico para todo el personal que interviene. Los hospitales que más refirieron pacientes fueron el IGSS, Roosevelt y San Juan de Dios en la ciudad capital, con un total de 50\% (13), siendo éstos hospitales grandes y contando con más personal especializado, el resto de hospitales que refirió pacientes son del interior del país, sin haber referido más de un paciente por cada centro asistencial, por lo cual se infiere que en el interior del país no se tiene a personal adecuado para la identificación de tales casos y así mismo para su oportuna referencia.

De los 26 pacientes, a 17 pacientes se les realizó switch arterial procedimiento de elección pues presentaban transposición de grandes arterias con septo integro, además que a 1 paciente también se le realizó corrección del arco aórtico por hipoplasia a quien se le llevó a arresto circulatorio, lo que también representó un reto importante para todo el personal que intervino, dicho paciente falleció. En la literatura se describe que el switch arterial se realiza de acuerdo a la anatomía cardíaca y las condiciones clínicas del paciente, sin embargo vale la pena mencionar que aun hoy por hoy en Guatemala las condiciones clínicas generales de los pacientes al momento de su evaluación inicial en UNICAR predisponen a realizar procedimientos paliativos pre switch arterial o bien, realizar el procedimiento correctivo (switch arterial) en pacientes con muy malas condiciones clínicas preoperatoria que influyen de manera determinante en los resultados del procedimiento quirúrgico. A 4 pacientes se les realizó, además del switch arterial, corrección de la comunicación interventricular que presentaban, lo cual da un punto más en la escala de estratificación de riesgo Aristóteles básico, aunque no aumenta su nivel de complejidad. A 3 pacientes se les realizó procedimiento de Rastelli pues su presentación anatómica y clínica no permitía que se realizará switch arterial. A 2 de los pacientes se les realizó procedimiento de Senning. Esto concuerda con la literatura existente en donde se describe que en las últimas décadas el Switch arterial se ha convertido el procedimiento quirúrgico de elección para la corrección de la transposición de grandes arterias, pues este es un procedimiento correctivo anatómico y aumenta la sobrevida de los pacientes disminuyendo la morbilidad y pronostico a largo plazo.

A todos los pacientes se les sometió a circulación extracorpórea para poder realizar el procedimiento quirúrgico, con una media de tiempo en bomba de circulación extracorpórea de 116 minutos y de clampeo aórtico de 93 minutos. La media de estadía en la unidad de cuidados intensivos cardiovasculares pediátricos fue de 14 días y la media de estancia intrahospitalaria fue de 20 días.

De los 26 pacientes sometidos a cirugía, 3 pacientes fallecieron trans-operatoriamente y 5 pacientes fallecieron post-operatoriamente, con una mortalidad global de $31 \%$, la cual se reduce aún más si se toma en cuenta la mortalidad por causa cardiaca, la cual es de 19\%, evidenciando disminución en comparación con los datos presentados en el año 2010 por el Dr. Leon-Wyss, quien reporto mortalidad del $47 \%$ en el tratamiento quirúrgico de la transposición de grandes arterias en UNICAR.

Aunque la mortalidad en unidades de cirugía cardiovascular pediátrica en países desarrollados ha sido reportada alrededor del 3-4\%, los países en vías de desarrollo con unidades de cirugía cardiovascular pediátrica con alto volumen quirúrgico (por ejemplo India) han logrado disminuir su mortalidad hasta 10-15\%. Programas, muchos de estos, hasta con tres veces más años de experiencia que la unidad pediátrica de UNICAR. Lo anterior evidencia que ha habido un avance significativo en la disminución 
de la mortalidad del tratamiento quirúrgico de los pacientes con trasposición de grandes arterias en Guatemala, resultado de la experiencia adquirida por el equipo multidisciplinario (cirujanos, intensivistas, anestesiólogos y perfusionistas) encargados de dar tratamiento a dichos pacientes. Lo anterior representa un reto importante a nivel institucional para Guatemala para así disminuir la mortalidad quirúrgica de los pacientes con cardiopatías congénitas, específicamente de los pacientes con transposición de grandes arterias.

Agradecimientos: el anterior trabajo se realizó gracias al apoyo del Dr. Iván Vides Ruiz, cirujano cardiovascular pediátrico de la unidad de cirugía cardiovascular de Guatemala; a la sección pediátrica de la unidad de cirugía cardiovascular de Guatemala, al Ing. Christian Edwin Sosa Sancé como revisor; y a los integrantes del organismo coordinador de trabajos de graduación de medicina.

\section{Referencias}

Dip, M; Halac, E; Cervio, G; Rojas, L; Bianco, G; Imventarza, O; Rodríguez-Bruno, S. (2011). Escala de riesgo quirúrgico en cirugía pediátrica (en línea). Cirugía Pediátrica 24(1):30-37. Consultado 05 feb. 2019. Recuperado de https://www.secipe.org/coldata/upload/revista/2011_24-1_30-37.pdf

Leon-Wyss, J; Lo Rito, M; Barnoya, J; Castañeda, AR. 2011. Persistent institutional difficulties in surgery for transposition of the great arteries in Guatemala: analysis with the Aristotle basic and comprehensive scores (en línea). World Journal for Pediatric and Congenital Heart Surgery 2(3)346-350. Consultado 17 feb. 2019. https://doi.org/10.1177/2150135111406291

Ronderos Dumit, M. 2010. Historia clínica en el paciente cardiópata (en línea, libro electrónico). In Cardiología pediátrica práctica. Ronderos Dumit, M; Palacio, G; Gutiérrez De Piñeres Rocha, O (eds.). Bogotá, Colombia, Editorial Distribuna. p. 30-34. Consultado 1 mar. 2019. Disponible en https:// booksmedicos.org/cardiologia-pediatrica-practica/

Vélez Moreno, JF; Echeverri Leal, ML. 2007. Transposición de grandes arterias (en línea). In Texto de cardiología. Charria García, DJ; Guerra León, PA; Manzur Jattin, F; Llamas Jiménez, A; Rodríguez Guerrero, NI; Sandoval Reyes, NF; Melgarejo Rojas, E (eds.). Colombia, Sociedad Colombiana de Cardiología y Cirugía Cardiovascular. p. 1361-1365. Consultado 23 ene. 2019. Disponible en http://www.bdigital.unal.edu.co/43639/37/cardiologia\%20Dr\%20Diaz.pdf

\section{Sobre el autor}

\section{Eliú Carlos Rafael Hernández Cordón}

Es Médico y Cirujano egresado del Centro Universitario de Oriente Cunori de Chiquimula. Residente de Cirugía, Hospital Roosevelt de Guatemala. 


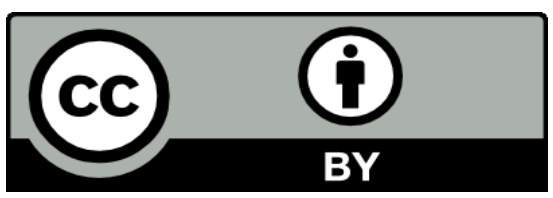

Este texto está protegido por una licencia CreativeCommons 4.0.

Usted es libre para compartir, copiar y redistribuir el material en cualquier medio o formato y adaptar el documento, remezclar, transformar y crear a partir del material para cualquier propósito, incluso comercialmente, siempre que cumpla la condición de atribución: usted debe reconocer el crédito de una obra de manera adecuada, proporcionar un enlace a la licencia, e indicar si se han realizado cambios. Puede hacerlo en cualquier forma razonable, pero no de forma tal que sugiera que tiene el apoyo del licenciante o lo recibe por el uso que hace. 